\title{
ELECTROPROTIDOGRAMA EN LA DISTROFIA CON EDEMAS (Estudio de 43 casos)
}

\author{
DrS. FLORENCIO A. BOLLO ' y RAMON MONTERO SCH, "
}

Con el objeto de precisar las alteraciones de los prótidos séricos en el curso de los trastornos nutritivos crónicos del lactante, en especial en aquellos que se acompañan de edemas y cuya denominación es variable: Distrofia Farinácea, Kwashiorkor, sindrome pluricarencial, pelagra infantil, etc. 's.s.12.14.1!.:!3, hemos recurrido a su estudio mediante la electroforesis en papel. Este método, como es sabido, consiste en la migración catódica $\mathrm{y}$ anódica que experimentan las particulas albuminoideas, ya sean del plasma o suero sanguineos, exudados, L. C. R., orina, leche, etc., al ser colocadas dentro de un campo

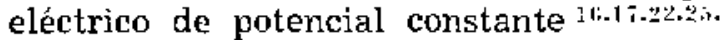
$36.40 .43-46$.

\section{METODO Y MATERIAL DE ESTUDIO}

Para nuestro estudio nos hemos servido del aparato conocido por Elphor $\mathrm{H}$, o método de Grassmann-Knedel-Hannig 14.25-1:;, sistema que no nos informa como otros más perfeccionados, sobre el fibrinógeno del plasma ni sobre las fracciones globulínicas gamma $2 \mathrm{y}$ gamma 3 ; pera que, sin embargo, dada su sencillez y reducido costo resulta de valor en la práctica clínica.

Desde ya, podemos informar que en ninguna de nuestras determinaciones encontramos fracciones Beta 2, o de paraproteínas.

El tiempo que hemos empleado en la electroforesis fué de 14 horas en un ambiente fresco ide 18 a $20^{\circ} \mathrm{c}$. I. Como colorante usamos con éxito el azul de Bromofenol en lugar del Negro Amidado, en atención a su menor costo e iguales resultados. Para la lectura de las bandas se empleó el densitómetro Elphor $\mathrm{H}$, previa colocación de estas en solución de Transparenzol. Por no disponer aún del Fotómetro automático Elphor-Integraph, con el que esperamos contar en breve, las

\footnotetext{
1 Becario argentino.

2 Cacedra de Podianria Pró. Anibal Arizita A.
} Hospital "Luis Calvo Mackenna". Servicio Nacional de Salud. áreas correspondientes a cada fracción fueron medidas por planimetría. En la representación gráfica de los resultades, hemos seguido el método de la Clínica Infantil de Münich expuesto por los Drs. Freislederer y Pache ${ }^{1}$.

Como escalia de valores normales usamos la dada a conocer por el Prof. H. J. Antweiler de Bonn Alemania ":, por considerar que aún no poseemos en la literatura nacional o Sudamericana, la suficiente casuística para las edades normales infantiles. No por ello dejamos de reconocer el esfuerzo que han significado los trabajos realizados en 1951 con el método de Tíselius, por los Drs. Werner Busta" mante, Manuel Madrid y Sergio González ${ }^{1 ;}$ y los efecutados recientemente por el Dr. Fernando Mönckeberg y colaboradores ${ }^{44}$, con el mismo sistema empleado por nosotros.

El cuadro No 1 muestra el tipo de protidograma, igual a cualesquiera de los 140 que se efectuaron para el presente trabajo.

El cuadro N 2 muestra las edades y el sexo de 43 niños afectados de Distrofia con Edemas, hospitalizados en los diferentes Servicios de Medicina del Hospital "Luis Calvo Mackenna" en el periodo 1955-56.

En todos los niños el primer protidograma se hizo al momento del ingreso y los siguientes con 10 días de intervalo, hasta completar en la mayoría de ellos, 3 a 4 determinaciones. En algunos pacientes, cuya hospitalización se prolongó, fué posible completar hasta 6 protidogramas. (En total, como ya dijimos se efectuaron 140 determinaciones electroforéticas).

$\mathrm{El}$ cuadro $\mathrm{N}^{\mathrm{o}} 3$ muestra el poreentaje medio de los protidogramas de ingreso de las 43 observaciones.

El cuadro Nu 4 muestra 36 protidogramas de 12 observaciones i con 10 dias de intervalo cada unal de niños afectados de Distrofia con edemas, de edad y condiciones estadisticamente semejantes (Observaciones correlacionadas ?. 


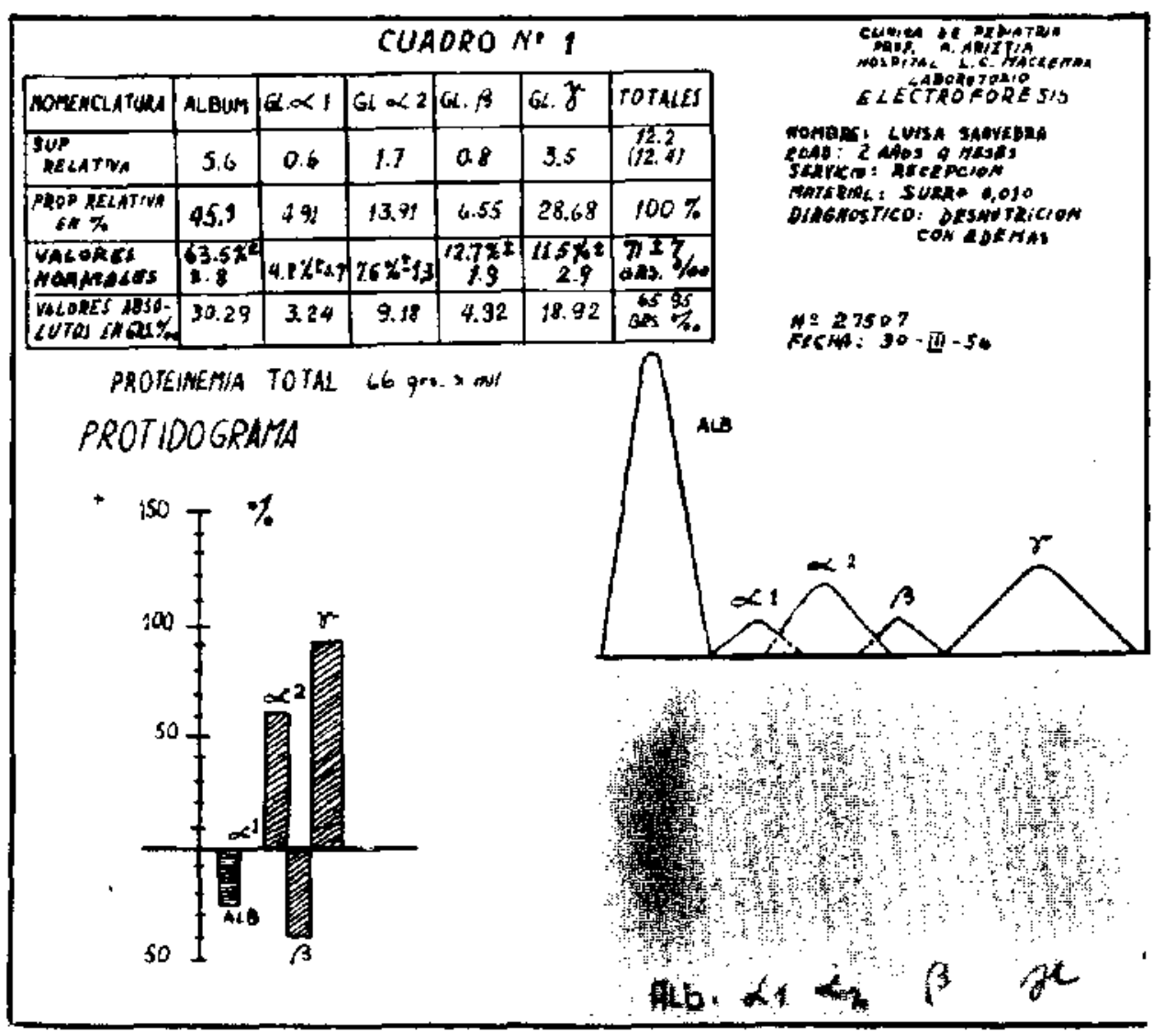

En este cuadro puede verse, comparando los protidogramas I-II y III, cómo se normaliza la cantidad y calidad de los protidos del plasma sanguíneo por la acción de la dietética $\mathrm{y}$ medidas higiénicas en el nin̄o hospitalizado. Estos niños no recibieron plasmoterapia ni transfusiones sanguíneas. A los 30 días de tratamiento el protidograma está en los límites de la normalidad.

Resumen y Comentario: Se estudian las alteraciones de los prótidos séricos por medio del electroprotidograma en 43 ni-

CUADRO NO 2

43 OBS. DE DISTROFIA CON EDEMAS SEGUN SGX" Y EUADES

\begin{tabular}{|c|c|c|c|c|c|}
\hline \multirow{2}{*}{$\begin{array}{c}\text { SEXo } \\
\ldots\end{array}$} & \multicolumn{4}{|c|}{ EUAI (meses) } & \multirow[t]{2}{*}{ TOTAL } \\
\hline & 6 a 12 & $13-18$ & $19-24$ & $25-30$ & \\
\hline MASCULINO & 4 & 8 & 4 & 1 & 17 \\
\hline FEMENINO & 5 & 7 & 7 & 5 & 26 \\
\hline TOTAI, & 12 & 15 & 12 & 6 & 43 \\
\hline
\end{tabular}

nos afectados de Distrofía con Edemas, hospitalizados en el Servicio de Medicina del Hospital de Niños "Luis Calvo Mackenna" durante los años 1955-56 y cuyas edades fluctuaban entre 7 y 30 meses.

El electroprotidograma de ingreso de las 43 observaciones reveló una disprotei-

$$
\text { CUADRO NP } 3
$$

RLECTROPROTODIGRAMA IDE INGRESO EN AR NINOS AFECTAOOS DE MSTROFIA CON EUEMAS

\begin{tabular}{|c|c|c|c|}
\hline & CENTA & LEDIO & VALORES NORMALES \\
\hline SEI & AS & $43,13 \%$ & $63,5 \% \pm 2,8$ \\
\hline & $r i$ & $5,64 \%$ & $4.8 \div 0.7$ \\
\hline 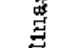 & $O_{-1}$ & $14,87 \div$ & $7,5 \% \pm 1.9$ \\
\hline$\frac{5}{0}$ & $\beta$ & $10,54 \%$ & $12,7^{\prime} ; \pm 1,9$ \\
\hline 0 & $Y$ & 22,75 & 11,$5 ; \pm 2.9$ \\
\hline $\mathrm{PRC}$ & DIO & & \\
\hline $\begin{array}{l}\text { PRO } \\
\text { Tota }\end{array}$ & $\begin{array}{l}\text { EMIA } \\
\text { s. (s) }\end{array}$ & 48.4 вть & \pm 7 grrs. \\
\hline
\end{tabular}


CUADRO No 4

ELECTHOPROTJDOGRAMAS EN 12 OBSERYACIONES CORRELACIONADAS DE NINOS AFECTADOS DE DISTROFIA CON RDEMAS

I II III

\begin{tabular}{|c|c|c|c|c|c|}
\hline & & PORCHATAUE & MEDIO & & VALORES NORMALES \\
\hline \multicolumn{2}{|c|}{ SERINAS } & $43,13 \%$ & 30,82 & $52,60 \%$ & $63,5 \% \pm 2,8$ \\
\hline \multirow{4}{*}{ 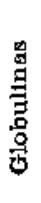 } & $\boldsymbol{a}_{\mathrm{t}}$ & $6,43 \%$ & $4.53 \%$ & $2+72 \%$ & $4,8 c_{i}^{\prime} \pm 0,7$ \\
\hline & $\alpha_{2:}$ & $14,82 \%$ & $19,79 \%$ & $12,55 \mathrm{r}$ & $7.5 \%=1,3$ \\
\hline & $\beta$ & $12 \%$ & $11,02 \%$ & $12,01 \%$ & $12,7 \% \pm 1,9$ \\
\hline & $\gamma$ & $23,6 \%$ & $18,56 \%$ & $14,87 \%$ & $11,5 \% \pm 7,9$ \\
\hline \multicolumn{2}{|c|}{$\begin{array}{l}\text { PROTIDEMIA } \\
\text { Total/grs, 尔 }\end{array}$} & 47,61 & 59,67 & 62,46 & $\pm 7 \mathrm{grs}$. \\
\hline
\end{tabular}

nemia, con marcada hipoproteinemia e hiposerinemia, aumento relativo y absoulto de las globulinas Alfa 2 y Gamma y niveles prácticamente normales de Alfa 1 y Beta globulinas.

De los 43 casos se excluyeron los que presentaron enfermedades infecto contagiosas o que recibieron plasmoterapia o transfusiones sanguíneas, ellos serán motivo de otras comunicaciones. Se formo con los restantes un grupo homogéneo de 12 casos correlacionados con 3 determinaciones cada uno: al ingreso, a los $10 \mathrm{y}$ a los 20 días de hospitalización.

En este grupo todos presentaron, en el primer protidograma, las características ya mencionadas. (Véanse cuadros $N^{9} 3$ y 4$)$.

En la segunda determinación, efectuada 10 días después (niños con régimen alimenticio sin carencia alguna, y que correspondió a la fase clínica de desaparición del edema, se observó una evidente mejoría de la disproteinemia: $1^{\text {g) }}$ ) proteinemia total prácticamente dentro de los límites normales, $2^{\circ}$ ) serinemia en vías de corrección, $3^{\circ}$ ) gamma globulina prácticamente sin modificaciones en relación al 1.er Protidograma.

En el tercer protidograma, a los 20 días de hospitalización observamos la disproteinnemia en franca recuperación: I) proteinemia total normal, II) hiposerinemia moderada, III) gamma globulina normal y Alfa 2 globulina medianamente elevada :

\footnotetext{
- Aunque por todas las determinaciones de esta última globnlina, hechas es el pais, esta cifra sería de absolute normalidad.
}

Con los resultados obtenidos en este estudio, consideramos que el electroprotidograma es de mucha importancia para valorar, conjuntamente con los demás signos clínicos, la intensidad, evolución y pronóstico del cuadro patológico conocido con la denominación de Distrofia con Edemas, pues nos informa de las modificaciones que las albúminas del órgano líquido de la economía (el plasma sanguíneo) experimentan en este cuadro de tan grave desnutrición.

\section{SUMMARY}

In Hospital Calvo Mackenna, in 1955 and 1956 , it was studied the serum protein in 43 infants (fron 7-30 months old) that suffered distrofia with edema by electroprotidograma.

The electroprotidograma taken at the ingress of these infants, showed a disproteinemia and hiposerinemia; an increasement of alfa 2 and gamma globulins and normal levels of alfa 1 and beta globulins.

The evolution of these examinations showed in twelve similar cases, an improvement, at first of the total proteinemia. second of serinas, then gamma globulin and at last, the rest of globulin fractions.

\section{BIBLIOGRAFÍA}

1.-HUNGRRLAND, HETNz. - Acerca de1 edema del niño prematuro. Revleta Española de Pedlatria. 10:819. 1954 .

2. COMEz, F. y Cols. - Estudios en n!ños desnutridos. Aunerican Journal of Diseages of Children. 87: 073.1054

3.-FRIRD, CH. y Cola. - Flpogamaglobultnemla con edema e hipoproteinemla. Pedlatrics, 4:59, 1954.

4.-Prot. E. ROMnNGTR, - vitimin T Goetsch. Archlv. rile Kindethellkunde. 40:1 1954. 
5.-LAPIN, J. H. - Errores comures en la alimentsclon infantl, The Journal of Pedlatitcs. 45:583, 1854.

6. STURE RAFSTEDT. - Studies on Serum LipIas and Lipoproteins in Infancy and Chlldhood. Acte PaeAiatrice. 44:588, 1955 .

7. STURE RAFSTEDT. - Studles on Berum Lipids and Llpoprotelns in Imrancy and Childhood. Acta Predtatrice. (Scandinatica). 44:102, 1955.

8.-B. C. TROWELL; J. N, P. DAVIES; R, F, A. DEAN Kwashtokor and Malnutrition. Acta Paecllatrica (Scandinavice). 44:487, 1955.

9.-GREENSPAN, E. M. and FREILING, D. A. - Serum mucoprotein level in differentiatin of hepatogenlo from obstructive jaundice. Arch. Internal Med, 91 474, 1953 .

10.-ROMArI, J. D. - La recherche e l'evaulacion des aLcoproteins de serum a l 'aide de I'electrophorese su papler, La Presse Medtcale. 62:178, 1954.

11. - G. D. PACHE und W. FREISLEDERER. - Beste hen Bezlebungen der Purpura Schöenleln Henoeh und der Periarterltis nodos zum rheumatischen Formenkreis? Monatsschrift für Kinderhellkunde iMünchen). 103:123.

12.-ARIZTLA. A. - sindromes edematosos con hipoprotelnemia en el niro menor, Rep. Chilena de Pect. $9: 1.1938$.

13.-ARIZTIA. A. - Trastornos nutrittros crónlcos en el lactante. Rev, Chtlena de Ped, 12:247, 1941.

14.-SCROGGIE, A. - sindromes policarenclales en $1 \mathrm{a}$ Infancla. Rev. Chllena de Ped. $13: 945,1942$.

15.-BUSTAMANTE, W.i MADRID. M. 5 GONZALES, S. Determinacion electroforétjea de las seroproteints sanguineas en el Lactante Eutrófico y Distrófleo. In Congreso Sudamericano de Ped. Montepldeo, 2: $283 \mathrm{y} 286,1951$.

16.-MONTERó, $R$.- La Electroforesis (sus fundamentor). Rev. Chilena de Ped. $27: 27,1955$.

17.-MONTBRO, R. - La electroforesle cuantitatlva, Rev. Chllena de Ped. $27 ; 82,1955$.

18-D CONDRA J - Distrofia plurlcarencial hidropigent. J, de Pedlat. 29:144, 1954.

19. GARRAHAN, J. - Comentario crítico sobre trastornos nutritivos del lactante y discrofia carenctal. Arch. ArR, de Ped. 37;121, 1952.

20. - Garratan y Cols. - Comentarto estadistteo sobre distrofia carenctal. Arch. Arg. de Ped, 37: 197, 1952 .

21.-MARTIN DU PAN, R. y Cols, - Estudio electroforético de las proteínas sericas en el prematuro durante log 4 primeros afios de la vida Arch. Franc. de Ped. 10:1013, 1953.

22.- ORLANDINI. O. $y$ Cols. - Las seroproteínas en el juctante en especial relación a la garna globulina. kev. Peruana de Ped. 11:99, 1952.

23.-NIEMEYRR, H.: LOPEZ, R. y MENEGFELLO, J. La hemodilución como factor coadruvante en el descenso de las proteinas serlcas en el edema de hambre, Rev. Chlleua de Ped. 21:157, 1949.

24,-ROLF CARBALLO. - Dlaproteinemias. (España). 1953.

25.-H, J, ANTWEILER. - Die Quantitave Elektmopho- rese in der Medizin. iBonn), Springer Verla-BerIn Gijttingen-Helderberg, 1952.

26.-H. KELLNFR und H., SUDHOF. - Monatsschrlft fur Kinderhelkunde. Zur chembchen Charakterisierumg des Sernmelwelsses. 103 Band. Heft 8-Auqust ". Päg. 385

27.-L.. KúliN. - Annales Faedlatrle1, sept. 34, Vol. 183. Fä. 162.

28.-J.. HOLMOLKA - Annales Paedlatricl. Agosto 5A. Vol, 193. Pág. 96,

28-G, HOOFT y $R$ CLARA - Annales Paedlatric1. Agosto 54. Vol. 182. Pag. 334.

30.-H.. SCHONENBERG. Annkles Paedatrici. Agosto 5 Vol, 182 . Phé, 355 .

31.-1. CORBkes y Cols. - Archlves Francelses de pe. dtatrle. DIC. 1954. Vol. 11. Pëg. 561.

22. C ANORA - Distrofta pluricarenclal hldropisena. Journal de Pedlatria. Abrli-Mayo, 54. Vol. 19 Pát. 144

33.-FRAY BENTOS $y$ YOUNG. - Resuitados $y$ experiencias con lipotróplcos en Cl. Pedlatrleas. Archlvos de Pedlatría del Jruguay, Vol, 25. Nov, 54. Pag. 727.

34. CAMACHO GAMBA. J. - Problemas de la desmutrlclón en los patses latimoamertcantos. Ei hierto endotenosos en la Anemla Carencial del Tróp1co. Revista Colomblana de Pedlatria y Puericultura. 14:81 1955 .

35-CROCCO, G y COLHITA A - Rlcerche sul contenute in glicoprotelne del siera nellinfanzia in condizione normali e patologiche. La Pedintrita. 9: 764,1953 .

36.-HOMOLKA, J. - Die Blutelwesskirper und thre veranderungen in der elektrophoretisch-polarographls chen Forschuag. Annales Prediatrici, 183:96, 1954.

37.-3CHONENBERG $\mathrm{Y}$ - Essentielle Hrpoprotelnamle mit Tetanta. Annales Paediaticl. 182:355. 1954.

38.-HOMOLKA, J, and MYDLIL. V. - Blood proteins in infants from a quantitatlve and qualttatlye polnt of view. Annales Prediatrici. 85:129. 1955.

39.-TUÑon, R. - Sobre tin caso de Kwrshlorkor. Acte Ped. Española. 152;608, 1955.

40-BLAvCO, M. Y PINTO, J. - Valor práctlco de Ia electroforesls en Pedfatria. Acte Ped. Esparioln, 153 631.1955

4L-TARACEN A, B. - Lr desnutmolón en el niño. Aete Ped. Espariola. 152:836. 1955

42.-ABALLI A, * Cols. - Alteraciones hepáticas en los trastornos nutritivos. Reviste Cubane de Pedatria. $26: 431,1954$.

43.-WUNDERL $\mathbf{Y}, \mathrm{CH},-$ Dle Papieretektrophorege. Verlag $B$. R. Saurlander y Co, Aarau y Frankfurt A. M. 44. -IVANOVIC - F MONCKEBERG Y M. PERRETTA. Estudilos de las proteinas sericas por electroforesis en papel. Tesis, 1956 .

45. CARMEN VKLASCO, GABRIEL LOBO PARGA $y$ Cols. Mtcroelectroforegis en papel de IAs Proteinas dol Suero del R. N. P lactarites normales. Rev. Chilena de Pediatria. 27: 1956.

46.-AGOGTINI $R$, y $M$, J, RUSSO, -- Electroforesis. Fracionatnlento nroteico en papei de flltro. Medictna Papamerlcenn. $6: 6,1956$. 